\title{
A Multivariate Approach to Ensure Statistical Reproducibility of Climate Model Simulations
}

\author{
Salil Mahajan \\ Oak Ridge National Laboratory \\ Oak Ridge, Tennessee, USA \\ mahajans@ornl.gov
}

\author{
Katherine J. Evans \\ Oak Ridge National Laboratory \\ Oak Ridge, Tennessee, USA \\ evanskj@ornl.gov
}

\author{
Joseph H. Kennedy \\ Oak Ridge National Laboratory \\ Oak Ridge, Tennessee, USA \\ kennedyjh@ornl.gov
}

\author{
Min Xu \\ Oak Ridge National Laboratory \\ Oak Ridge, Tennessee, USA \\ xum1@ornl.gov
}

\author{
Matthew R. Norman \\ Oak Ridge National Laboratory \\ Oak Ridge, Tennessee, USA \\ normanmr@ornl.gov
}

\begin{abstract}
Effective utilization of novel hybrid architectures of pre-exascale and exascale machines requires transformations to global climate modeling systems that may not reproduce the original model solution bit-for-bit. Round-off level differences grow rapidly in these non-linear and chaotic systems. This makes it difficult to isolate bugs/errors from innocuous growth expected from round-off level differences. Here, we apply two modern multivariate two sample equality of distribution tests to evaluate statistical reproducibility of global climate model simulations using standard monthly output of short ( 1-year) simulation ensembles of a control model and a modified model of US Department of Energy's Energy Exascale Earth System Model (E3SM). Both the tests are able to identify changes induced by modifications to some model tuning parameters. We also conduct formal power analyses of the tests by applying them on designed suites of short simulation ensembles each with an increasingly different climate from the control ensemble. The results are compared against those from another such test. These power analyses provide a framework to quantify the degree of differences that can be detected confidently by the tests for a given ensemble size (sample size). This will allow model developers using the tests to make an informed decision when accepting/rejecting an unintentional non-bit-for-bit change to the model solution.
\end{abstract}

\section{CCS CONCEPTS}

- Software and its engineering $\rightarrow$ Software verification and validation; Empirical software validation.

\section{KEYWORDS}

Reproducibility, climate models, multivariate statistics

\section{ACM Reference Format:}

Salil Mahajan, Katherine J. Evans, Joseph H. Kennedy, Min Xu, and Matthew R. Norman. 2019. A Multivariate Approach to Ensure Statistical Reproducibility of Climate Model Simulations. In Proceedings of the Platform

Publication rights licensed to ACM. ACM acknowledges that this contribution was authored or co-authored by an employee, contractor or affiliate of the United States government. As such, the Government retains a nonexclusive, royalty-free right to publish or reproduce this article, or to allow others to do so, for Government purposes only.

PASC '19, June 12-14, 2019, Zurich, Switzerland

(c) 2019 Copyright held by the owner/author(s). Publication rights licensed to ACM ACM ISBN 978-1-4503-6770-7/19/06 ..\$15.00

https://doi.org/10.1145/3324989.3325724 for Advanced Scientific Computing Conference (PASC '19), June 12-14, 2019, Zurich, Switzerland. ACM, New York, NY, USA, Article 4, 10 pages. https: //doi.org/10.1145/3324989.3325724

\section{INTRODUCTION}

The US Department of Energy's (DOE) Energy Earth System Modeling (E3SM) project [2, 4] is mandated to utilize leadership class high performance computers effectively as they advance towards exa-scale, to simulate the fully coupled earth system climate. The nature of computer architecture layouts of current and future DOE machines encourage algorithms and code structure that result in solutions with round-off differences. Round-off differences in finite precision arithmetic in parallel applications are generally caused by the order of a sequence of computations, which may depend on the order of messages arriving from different parallel processes. These can occur due to change in compilers, compiler options, machine architecture, numerical schemes, implementation of sub-grid scale physics, etc. In climate simulations, which are weakly forced and chaotic like the climate system itself, these differences produce different weather over time. Model verification of the long-term climate is therefore challenging even after minor changes to software, programming environment, or hardware; these round-off differences grow rapidly.

To assess the impact of round-off differences on the climate simulation, one approach has been to test whether the differences in atmospheric temperature between a perturbed and a control run during the first few simulated days are within a tolerance level [13]. This tolerance level is estimated by quantifying the growth of small perturbations of the initial conditions in the non-linear system. However, atmospheric parameterizations (sub-grid scale process representations) are ill conditioned, and increasingly becoming more so as they become more complex, such that small changes in their input lead to large differences in their results [1]. Thus, small perturbations accumulate rapidly and exceed the tolerance thresholds within the first few time steps, which renders model verification within a few model days almost impossible. This makes it increasingly difficult to identify possible systematic bugs/errors in non-bit-for-bit (non-bfb) reproducible changes to the model from growth of round off level differences. Recent work is being directed at modifying these parameterization routines to prevent such behavior. Nonetheless, these perturbation growth tests do not target the reproducibility of the full model. 
The most straightforward alternative is to measure whether the long-term climate statistics of the perturbed and control run are similar [13]. Model verification in this case requires the integration of the perturbed model for a long simulation period, over which perturbations from parameterizations become less relevant. To determine the effect of model variations at a given scale, a run length sufficient to capture that variability is required (for example, multidecadal simulations are required to assess El Niño Southern Oscillation (ENSO), which has a time period of 2-7 years). Then, the mean and variable climate state of the simulation is compared against observations and/or a previously accepted control run. In the climate community, this is fulfilled by a subjective comparison of the long perturbed simulation against a baseline simulation of hundreds of years by experts who systematically browse through a large set of diagnostic plots and tables. High-resolution global simulations are costly; a typical 10-year simulation of the production resolution E3SM (v1) has a throughput in the order of weeks, including time spent in computing queues, and costs about $15 \mathrm{~K}$ core hours/simulated year. Thus model verification of this type is painstakingly slow and expensive, and it is still an incomplete process that lacks depth and objectivity. With a large number of software and scientific code changes currently underway to move beyond the first release of E3SM, relying on expert advice for each minor code change is unrealistic and therefore will be avoided altogether.

To ensure proper model porting to new machines and compilers, Baker et al. [1] proposed a principal component based testing approach where they run a large ensemble ( $>150$ members) of short (1 yr simulation length) control simulations to derive the statistical properties of model output of the simulation ensemble. They then compare a small number (about 3) of short simulations with the ported model to evaluate if the statistics (global annual average of output variables) of the ported model simulation are significantly different from that of the large control ensemble. Mahajan et al. $[7,8]$ applied a testing approach where they also compare short ( $1 \mathrm{yr}$ ) ensembles (about 30 members) of the control as well as the perturbed simulation to evaluate if the climate statistics of the two ensembles are statistically similar by evaluating the global annual average of each model output variable separately. Massonnet et al. [9] compare the distribution of a normalized index of 13 variables to evaluate the reproducibility of a fully coupled model using 5member ensembles of $20-\mathrm{yr}$ simulations.

These short simulation ensemble members only differ in the initial conditions, each perturbed randomly with a machine precision order difference at each grid point in the three dimensional temperature field. The chaotic nature of climate ensures that the ensemble simulations with differing initial conditions diverge quickly and become independent of each other in about 3-4 weeks of simulation length [e.g. 8] exhibiting different weather but similar climate statistics. The spread of these independent simulations thus represents the noise statistics (natural variability) of the system on these short time-scales. Also, climate model simulations have high latency costs that increase at scale - the workload per node reduces and the number of MPI messages increases but reduce in size as the model is scaled to greater number of compute nodes [8]. Thus, running a $N$ member ensemble as a single job is more efficient than running an $N$ year long run serially on the same number of nodes, because the workload on each node increases $N$ times, while also reducing communication latency.

Establishing the significance of the difference in the statistics of global annual average variables, as in the above approaches, can be framed as a multivariate two sample equality of distribution test, with a dimension size equal to the number of variables and sample sizes equal to the ensemble sizes. There are several modern powerful two sample equality of distribution tests that have been developed by the statistics and machine learning communities for multivariate distributions in recent years that are suitable for low sample sizes and high-dimensional data. Examples of such tests include the cross-match test [e.g. 12], energy test [e.g. 14], and the kernel test [e.g. 5] which are widely popular in other scientific fields. These tests can be exploited to compare climate model statistics as well. Here, we adapt two such tests (energy test and kernel test) for evaluating the statistical reproducibility of a climate model simulation after changes are introduced in the model. These tests will thus be useful in decisions involving approval of modifications (for example, a restructuring of some model source code to improve model performance or a change to its other software of hardware infrastructure) that result in a simulation that is not bit-for-bit reproducible. Here, we use equal ensembles $(>30)$ of the control and modified runs following Mahajan et al. [7, 8] and compare the climate statistics of the two ensembles using these modern multivariate tests.

In this study, as a step towards routine applications, we evaluate the robustness of these new tests by first applying them to test cases where a known climate changing behavior has been introduced, as in previous studies [1, 7, 15]. Following Mahajan et al. [7], we also conduct a formal statistical power analysis of these tests, using experimental suites where the climate statistics of the perturbed ensemble are controlled to be increasingly different, to determine the degree of difference that these tests can detect. We do not, here, apply the test to situations where the perturbed model is unavoidably non-bfb reproducible (for e.g. due to code refactoring) to evaluate if the climate simulation remains statistically reproducible. But, we foresee applying these tests to such scenarios in the near future.

\section{MULTIVARIATE TESTS}

We use two types of tests here, namely the energy test and the kernel test, to test the null hypothesis that the baseline (control) and the modified model simulation ensembles belong to the same population. These tests will be applied on short simulation $(\sim 1$ year) ensembles of the control and the perturbed model following Baker et al. [1] and Mahajan et al. [8]. Baker et al. [1] suggest that a simulation length of one year is sufficient for initial condition perturbation growth while also remaining computationally feasible. Also following Baker et al. [1] and Mahajan et al. [8], we use the global annual average of all the standard model monthly output variables (134 variables). We also compare the results from these tests against the existing Kolmogorov Smirnov (KS) testing framework $[7,8]$. The two new tests and the KS testing framework are briefly described below: 


\subsection{Energy test}

The energy test is based on the concept of energy statistics of Székely and Rizzo [14], where they define the test statistic e-distance, $e$, as:

$$
\begin{aligned}
& e=\frac{n m}{n+m}\left(\frac{2}{n m} \sum_{i=1}^{n} \sum_{k=1}^{m}\left\|X_{i}-Y_{k}\right\|-\right. \\
& \left.\frac{1}{n^{2}} \sum_{i=1}^{n} \sum_{j=1}^{n}\left\|X_{i}-X_{j}\right\|-\frac{1}{m^{2}} \sum_{l=1}^{m} \sum_{k=1}^{m}\left\|Y_{l}-Y_{k}\right\|\right)
\end{aligned}
$$

where $X_{1}, \ldots, X_{n}$ and $Y_{1}, \ldots, Y_{m}$ are the multivariate vectors of the baseline and perturbed ensembles. Here, $X_{i}$ represents a vector with a size of 134 variables, representing global annual average means of the 134 variables in the standard model output, for ensemble member $i$. And, $n$ and $m$ refer to the ensemble size of the baseline and the perturbed simulation respectively. Large values of $e$ correspond to different distributions of the two samples. Here, $\|A-B\|$ represents some measure of distance between two vectors $A$ and $B$; we use the euclidean distance. The null distribution of $e$ is not distribution-free but a permutation test approach provides a distribution-free test: the data vectors are pooled together and randomly resampled into the two groups and the e-distance $e_{k}$ is computed for each such permutation, $k$. If all distinct possible permutations are drawn, then the permutation test is exact. The values of $e$ from these permutations then describe the null distribution of $e$. For, a significance level (Type I error rate or false positive rate - erroneously rejecting a true null hypothesis) of $a$, the null hypothesis is rejected if $e>100(1-a) \%$ of the permuted estimates of $e_{k}$. Here, we resample 500 times to derive an approximate null distribution of $e$.

\subsection{Kernel Test}

In a kernel two-sample test, a smooth function (kernel) is fitted to each of the multivariate vectors of the two ensembles such that the function values are large for vectors of one ensemble and small for the other [e.g. 5]. The test statistic is the distance between the mean of function values over the two ensembles, called the Maximum Mean Discrepancy (MMD). If the two ensembles belong to the same population then we expect small values of $M M D$. Functions in the reproducing kernel Hilbert space (RKHS) like the Gaussian and the Laplace kernels have favorable properties suitable for the kernel test [5]. Here, we use the Gaussian kernel. An empirical estimate of the test statistic is given by:

$$
\begin{aligned}
M M D & =\left(\frac{1}{n^{2}} \sum_{i, j=1}^{n} k\left(X_{i}, X_{j}\right)-\right. \\
& \left.\frac{2}{n m} \sum_{i, j=1}^{n, m} k\left(X_{i}, Y_{j}\right)+\frac{1}{m^{2}} \sum_{i, j=1}^{m} k\left(Y_{i}, Y_{j}\right)\right)^{\frac{1}{2}}
\end{aligned}
$$

where $k$ represents the kernel in its class of functions that maximizes $M M D$ and $X_{1}, \ldots, X_{n}$ and $Y_{1}, \ldots, Y_{m}$ are the multivariate vectors of the baseline and perturbed ensembles. The null distribution of $M M D$ has a complex form, but the permutation test approach, as described above for the energy test, is routinely applied to establish the null distribution of $M M D$ too. Again, here, we resampled 500 times to arrive at an approximate null distribution of $M M D$.

\subsection{Kolmogorov Smirnov (KS) Testing Framework}

The KS testing framework is adapted from Mahajan et al. [8] and Mahajan et al. [7]. Here, we largely use it for comparison with the results of the energy and kernel tests and briefly describe it here. In the framework, a null hypothesis is tested for each output variable independently. The null hypothesis is that the variable values from the perturbed run and the control run belong to the same population $\left(H_{0}\right)$. The non-parametric (distribution-free) KolmogorovSmirnov univariate test is used for testing this null hypothesis. The larger null hypothesis $\left(\mathbf{H}_{\mathbf{0}}\right)$ that the climate statistics are statistically equivalent between the two ensemble runs is then evaluated as the number of variables that reject the null hypothesis.

Again, the null distribution for this test statistic (the no. of variables that reject $H_{0}$ ) is derived using re-sampling techniques. Here, we use the bootstrap approach. From a large control run ensemble, ensemble members are drawn and pooled into two groups, sizes $n$ and $m$ respectively. The number of variables that reject $H_{0}$ are recorded for each such drawing. Here, we re-sample 500 times. The critical value for a given significance level for the null hypothesis is then derived from the distribution of the recorded data (test statistic). For example, the critical value was found to be 13 for a significance level of 0.05 for a control run ensemble of the F1850C5 compset. The results presented here are not affected if a permutation test approach, like the one used for the energy and the kernel tests described above, is used for re-sampling. Mahajan et al. [7, 8] also found that the bootstrapping approach and the permutation test approach yield similar results. The KS testing framework and the associated re-sampling approaches are outlined in greater detail in Mahajan et al. [7, 8] and we generally follow them here.

\section{TEST CASES: KNOWN CLIMATE STATISTICS CHANGING SIMULATIONS}

Following previous work [e.g. 1], we conduct short simulation ensembles by changing a model tuning parameter that is known to have a significant impact on the climate simulation $[1,7,15]$, to evaluate our tests. Table 1 lists three such separate ensemble simulations each with a different tuning parameter modified directly impacting different parts of the model. Table 1 also lists the default (control) and the modified parameter values for the three cases. These tuning parameters are a few among several that are routinely perturbed to adjust model behavior to match observational dataset and maintain the model's energy balance after any climate modifying changes to the model (e.g. introduction of a new parameterization scheme, change in model resolution) [e.g. 11]. Previous studies have used a larger set of experiments modifying several other tuning parameters. We plan to expand our testing suite of experiments to match those in the near future.

The three model tuning parameters used here directly impact different physical parameterizations of the model climate system. $z m_{-}$c0_ocn controls the rate of efficiency of the conversion of cloud 
droplets to precipitation in the deep convection scheme. The parameter $d c s$ controls the accretion rate of cloud ice to snow and impacts stratiform cloud microphysics. And, rhminl controls the formation of low clouds and represents the relative humidity threshold for the generation of low clouds and thus impacts cloud macrophysics properties, such as cloud fraction. Here, we use the ultra-low resolution version of E3SM with a horizontal resolution of about $500 \mathrm{~km}$, with the F1850C5 compset, which simulates the Earth system's pre-industrial climate of the atmosphere and land forced with bestestimate pre-industrial greenhouse gas, aerosols and ozone radiative forcing and prescribed with pre-industrial sea surface temperatures and sea-ice conditions. The same model configuration was also used by Mahajan et al. [7].

Here, we evaluate if the statistical tests could identify changes in the climate statistics as compared to the control model ensemble each with 30 members. Similar sets of experiments have been used by previous studies to establish the robustness of testing methodologies $[1,7,15]$. Table 2 lists the results of the null hypothesis test at the $95 \%$ confidence level for each of the three testing approaches. The approximate null distribution of the test statistic in each case is derived using resampling techniques as described in the previous section.

All three tests are able to reject the null hypothesis that the climate statistics of the two simulation ensembles are statistically different for all three experiment sets. For similar values of the ZM tuning parameter, albeit using different models and resolutions, Baker et al. [1], Wan et al. [15] and Mahajan et al. [7] using their principal component approach, time convergence approach and KS testing framework respectively, were able to identify that the climate simulation of the perturbed model is different from the control. Also, for similar values of the rhminl in the MACRO experiment, Baker et al. [1] and Wan et al. [15] were also able to identify a significant difference in the two models. The MICRO perturbed experiment, not conducted by these previous studies, is also known to have a significant impact on the model climate [e.g. 11], which the tests here flag. These results point towards the robustness of all three tests in identifying known climate changing behavior.

\section{POWER ANALYSIS: TYPE II ERROR RATE}

The above test results (Section 3, Table 2) only indicate that the statistical tests are able to catch certain differences in climate statistics with a significance level of 0.05 . However, they provide no indication on the magnitude of differences that these tests can detect at the given significance level and given sample size. Neither do they inform on the sample sizes needed to detect a given magnitude of difference. To answer these questions, we conduct a formal power analysis of these reproducibility tests using several additional simulation ensembles by modifying the three listed parameters in a controlled manner. Power analysis refers to the quantification of the Type II error rates $(\beta)$ of statistical tests, also referred to as the false negative error rate - the rate of falsely accepting a null hypothesis.

\subsection{Controlled Parameter Perturbation Ensembles}

We gradually change the value of a tuning parameter progressively increasing its difference from the default value. We conduct 100 member simulation ensemble for a series of discrete values within a specified range of the tuning parameter. Each of these short simulation ensembles have an increasingly different climate from the control ensemble (100 members) by design. We conduct such controlled experiments with all three tuning parameters separately as listed in Table 1 that we used for our test cases (Section 3). Table 3 lists the range of values of the tuning parameter values used to generate these simulation ensembles. The range of the parameter values used here lies within the range of plausible estimated values of these parameters [e.g. 11]. The ZM_SET is adapted from Mahajan et al. [7] and we use the same discrete values of $z m_{-} c 0 \_o c n$ here. The MICRO_SET and the MACRO_SET are new simulation ensembles, and here we present results of power analysis for all three tests for all three simulations ensembles. Mahajan et al. [7] conducted the power analysis of the KS testing framework only for the ZM_SET. Here, we extend that work to include power analysis of the KS testing framework for the MICRO_SET and the MACRO_SET.

\subsection{Deep Convection Term}

Fig. 1a shows the statistical power $(1-\beta)$ of the Energy test for the ZM-SET experiments, which was conducted as follows. For a sample size, $N(=M), N$ ensemble members are randomly selected separately from the 100 member control $\left(z m_{-} c 0 \_o c n=0.007\right)$ ensemble as well as from a 100 member perturbed tuning parameter ensemble (say $z m_{-} c 0 \_o c n=0.0075$ ). The Energy test is conducted on these pair of $m$-sized samples at the given statistical significance (0.05). The null hypothesis is that the two samples belong to the same population. This step is repeated $K(=500$, here $)$ number of times. The statistical power for a sample size $N$ at the given significance level represents the probability of the test successfully rejecting the null hypothesis - quantified here as the fraction of times the null hypothesis is rejected for the $K$ draws. Fig 1a thus shows the power of the Energy test for different sample sizes $(N=30,40,50,60)$ at detecting changes in the simulation as the zm_c0_ocn parameter value (and hence the simulated climate) increasingly differs from the control ensemble.

Fig. 1a shows that the statistical power of the test increases with an increase in ensemble size. For example, a change in zm_c0_ocn from 0.0070 to 0.02 is flagged as differing climate about $90 \%$ of the times with 60 ensemble members, whereas with 30 -member ensembles that probability reduces to about $20 \%$. The probability of detecting this difference increases for the 40- and 50-member ensemble sizes as compared to the 30 -member ensembles. Interestingly, for the test case ZM (Section 3, Table 1) the energy test correctly flagged the modified case with zm_c0_ocn $=0.045$ with 30 -member ensembles as statistically different. However, the probability of successfully identifying the change in climate is only about $60 \%$. The power analysis suggests that to successfully detect such a magnitude of change, with a greater than $95 \%$ probability, the ensemble size should be about 40 . To detect climate changing behavior smaller than the impact of changing $\mathrm{zm}$-c0_ocn from 0.0070 to 0.02 with a high probability (greater than $95 \%$ ), the ensemble 
Table 1: List of known climate changing experiments with control and modified parameter values.

\begin{tabular}{llllll}
\hline Experiment Name & Ens. Size & Parameter Name & Parameterization & Default & Modified \\
\hline ZM & 30 & zmconv_c0_ocn & Deep Convection & 0.007 & 0.045 \\
MICRO & 30 & dcs & Cloud Microphysics & 400.0 & 100.0 \\
MACRO & 30 & rhminl & Cloud macrophysics & 0.895 & 0.85 \\
\hline
\end{tabular}

Table 2: Results of the null hypothesis test for four known climate changing simulation ensembles using different testing approaches. The null hypothesis is that the perturbed and control climate belong to the same population.

\begin{tabular}{llll}
\hline Experiment Name & KS test & Energy test & Kernel test \\
\hline ZM & Reject $\mathrm{H}_{0}$ & Reject $\mathrm{H}_{0}$ & Reject $\mathrm{H}_{0}$ \\
MICRO & Reject $\mathrm{H}_{0}$ & Reject $\mathrm{H}_{0}$ & Reject $\mathrm{H}_{0}$ \\
MACRO & Reject $\mathrm{H}_{0}$ & Reject $\mathrm{H}_{0}$ & Reject $\mathrm{H}_{0}$ \\
\hline
\end{tabular}

Table 3: Tuning parameter range used for controlled parameter perturbation simulation ensembles

\begin{tabular}{llll}
\hline Experiment Name & Parameter Name & Default & Range \\
\hline ZM-SET & zmconv_c0_ocn & 0.007 & $0.0071-0.045$ \\
MICRO-SET & dcs & 400.0 & $100.0-397.0$ \\
MACRO-SET & rhminl & 0.895 & $0.80-0.8925$ \\
\hline
\end{tabular}

size needs to be larger than 60 . But, for changes larger than that, a sample size of 60 is sufficient.

Fig. $1 \mathrm{~b}$ shows the power analysis curve for the Kernel test. From comparing Fig. 1a and $1 \mathrm{~b}$ it appears that the kernel test exhibits slightly greater power than the energy test with all ensemble sizes able to detect smaller changes in the climate with a slightly higher probability. For example, for an ensemble size of 40 , the kernel test is able to detect changes in the climate induced by a change in zm_c0_ocn from 0.0070 to 0.02 by a probability of about $90 \%$ as compared to about $80 \%$ for the energy test. For the ZM test case (Table 1) with 30 member ensembles, where $\mathrm{zm}$-c0_ocn is perturbed from 0.0070 to 0.045 , the kernel test correctly identified the change in climate. The power of the test indicates that we can be pretty confident in this result (power $=0.9$ ).

Fig. 1c is reproduced from [7] for the KS testing framework. Comparing the three figures, the KS testing framework demonstrates the greatest power among the three tests at detecting small differences in climate. With an ensemble size of 30, one can confidently (greater than $95 \%$ probability) detect changes as small as that from changing $z m_{-} c 0 \_o c n$ from 0.0070 to 0.0074 with the KS testing framework. With an ensemble size of 60 , one can increase the power of detection of change in climate statistics induced by changes to $z m \_c 0 \_o c n$ as small as from 0.0070 to 0.00712 .

\subsection{Cloud Microphysics Term}

Fig. 2 shows the power analysis curves for the three tests for the MICRO_SET experiment where the dcs term is gradually modified from a default value of 400.0 to values resulting in an increasingly different climate. Both the energy and kernel tests exhibit similar power for dcs magnitudes differing from 400.0 to 325.0. For, smaller perturbations both tests have weak power, with the energy test exhibiting a little more power. In the future, we would conduct more ensemble simulations with $d c s$ magnitudes in the range of 350.0 to 325.0 , where there appears to be a jump in the power of both tests, to better quantify the power of the tests for $d c s$. The KS testing framework again appears to exhibit the greatest power (Fig. 2c). With an ensemble size of 30 or greater, the test is able to flag differences as small as between dcs values of 400.0 and 390.0 with almost $100 \%$ probability. With 60 ensemble members, the test detects differences as small as 400.0 to 395.0 for $d c s$. Between $d c s$ values of 397.0 and 390.0 the power of the test increases with ensemble size as compared to the default value of 400.0 .

\subsection{Cloud Macrophysics Term}

Power analysis curves for the MACRO_SET experiments where the tuning parameter $r$ minl is gradually reduced from its default value of 0.8975 are shown in Fig 3. Both the energy (Fig. 3a) and the kernel (Fig. $3 \mathrm{~b}$ ) tests demonstrate similar power, detecting changes induced when the rhminl parameter is reduced slightly to 0.87 or lower with almost $100 \%$ probability for all ensemble sizes. The power drops significantly for both tests at detecting changes induced by rhminl value of 0.88 for ensemble sizes of 30 and 40 . With an ensemble size of 60 , both tests can detect the change in climate statistics for rhminl $=0.88$ as compared to the default. Again, the KS testing framework (Fig. 3c) seems to exhibit the strongest power detecting a change in rhminl from 0.8975 to 0.895 with $>95 \%$ probability with an ensemble size of 60 . For smaller ensemble sizes (50, 40 and 30), the test can detect differences in climate induced by a change in rhminl from its default value to 0.8925 with $100 \%$ probability. 


\section{SUMMARY, DISCUSSION AND FUTURE WORK}

Here, we apply two modern non-parametric multivariate two sample equality of distribution tests, namely energy test and kernel test, to evaluate statistical reproducibility of climate model simulations post non-bfb reproducible changes. Although it needs to be clearly demonstrated, we foresee that such tests would be useful in identifying harmless round off level differences from systematic bugs that might have been introduced during changes to the code as it is adapted for high-performance hybrid architectures that unavoidably result in a non-bfb reproducible solution. Both the tests are able to identify changes in the model climate introduced by known climate changing perturbations in model tuning parameters, similar to other tests.

A formal power analysis of the tests indicates that they can detect even smaller differences than those induced by these test cases and the probability of detecting these differences increases with an increase in ensemble size. Based on analyses using three controlled parameter perturbation experiment suites, it appears that for the same sample size (ensemble size) these tests have lower power than the KS testing framework proposed by Mahajan et al. [7, 8], which is able to detect much smaller magnitudes of change. Also, a rejection of the null hypothesis by the energy and the kernel tests would indicate a larger change in the climate statistics. However, it is important to evaluate these tests on additional scenarios and test cases to establish individual tests or a combination of tests that would be most suitable for climate reproducibility.

Nonetheless, the formal power analysis - providing the magnitude of change the tests can detect - will allow model developers using these tests to make an informed decision when accepting/rejecting an unintentional non-bfb change to the model solution. In the future, we plan to expand these power analyses using experiments with controlled perturbations to other common model parameters further elaborating the capabilities of the tests to the benefit of integrators. In addition, it is important to understand the impact of using different normalizing/standardizing procedures to the multivariate climate model output variables to the results of these tests. Also, using different distance metrics for the energy test and different kernel functions for the kernel tests may also impact results. We plan to investigate these impacts in the future.

Using the KS testing framework Mahajan et al. [8] found that an aggressive PGI compiler optimization (-fast option), which is known to change numerical results [10], significantly changes the climate statistics at the $95 \%$ confidence level (or a false negative rate of 0.05 ). The above result may also suggest that the false negative rate of the test was set too high, if this optimization change is not expected to change climate statistics. The decision to choose an appropriate false positive rate for a null hypothesis test is typically based on the required sensitivity of the test, and this needs to be explored further in the context of climate reproducibility. Mahajan et al. [7] using the same test confirmed that the untouched F1850C5 compset from version 0 of the model was reproducible even with version 1 of the model despite numerous changes to software libraries, compilers, software infrastructure and changes to model source code - albeit external to the F1850C5 compset codes, during months of model development. Also, Baker et al. [1] have used their approach to identify issues with certain compilers on certain machines. Massonnet et al. [9] also applied their index based test as they ported their model to a new machine. We plan to apply the new multivariate tests for such real world scenarios in the near future, which will also help establish an optimum balance between the false positive and false negative rates of the tests.

Also, as tolerance based tests evolve to test for numerical reproducibility in a few model time-steps using a few variables, it will be interesting to learn about cases where each of the testing approaches is most suited. For example, non-bfb changes that impact temperature tendencies significantly would be detected by inexpensive tolerance based tests too. But, when the immediate impact is less tangible, the climate statistics based approach may be more effective. In addition, climate statistics based tests will be more suitable to detect errors that might occur in source code that is not activated within several time-steps. For example, in the infrastructure source code that are outside the time-stepping loop like domain decomposition, initialize and finalize statements, MPI commands or processing variables for output and writing output files.

The 1-yr long ensemble simulations capture the spread associated with rounding differences in the initial conditions. In the future, we would evaluate if shorter simulation lengths are enough to quantify natural variability of the system and could be used to identify possible difference in climate statistics. We would like to extend these tests to the ocean model as well in the future. The time-scale of oceanic/coupled phenomenon are long (e.g. ENSO) and thus it would be interesting to study if shorter simulations as used here could be used for non-bfb reproducibility testing.

In some instances, non-bfb changes are propagated into E3SM. These decisions are based on ad-hoc approaches. In the near future, we would like to apply these tests to these scenarios to ensure that these non-bfb changes still produce the same climate statistically. To facilitate this, we've begun developing these tests into EVV, a python-based verification and validation package for E3SM, which will run each of these tests on a control and test ensemble, return a reject/accept status for each test, and provide a detailed breakdown of each tests' results in a portable website that can be easily shared to communicate test results. EVV version 0.1 [6] provides the K-S test, while the energy and kernel tests are slated for version 0.2 . Furthermore, within E3SM's case control system CIME, a set of tests have been created which will automatically setup and run the requisite test ensemble and then launch EVV, pointing to previously stored control ensembles. This allows these tests to be used within the normal E3SM development workflow and analyze any non-bfb changes that arise.

In addition to code re-factoring, ongoing efforts to accelerate climate model simulations on new deep learning machines include artificial intelligence (AI) approaches. Deep neural networks (DNN) that emulate large software kernels and computationally expensive parameterization modules are being developed in an effort to replace them within E3SM and other climate models [e.g. 3]. For example, a DNN is being developed that emulates the radiation kernel in an embedded cloud resolving model version of E3SM. While the DNN reproduces the instantaneous computations of the radiation kernel with a 95\% accuracy, it is not clear if simulations that use DNN instead of the radiation kernel produce the same 
climate. Other studies that evaluate the usability of DNN within climate models currently employ generally ad-hoc approaches. Testing approaches, as studied here, that can confidently detect changes to model climate simulation would provide a useful tool in the systematic evaluation of AI approaches in climate modeling. In the near future, we plan to apply our tests to E3SM simulation ensembles using DNN vs. those using the traditional radiation kernel to establish if these are statistically equivalent.

\section{ACKNOWLEDGMENTS}

This research was supported as part of the Energy Exascale Earth System Model (E3SM) project and Climate Model Development and Validation program, funded by the U.S. Department of Energy, Office of Science, Office of Biological and Environmental Research. This manuscript has been authored by UT-Battelle, LLC and used resources of the National Center for Computational Sciences at Oak Ridge National Laboratory, both of which are supported by the Office of Science of the U.S. Department of Energy under Contract No.DE-AC05-00OR22725. E3SM simulation data output is archived at U.S. Deparment of Energy's leadership computing facilities and can be obtained by contacting the corresponding author. This work used the R package 'energy' and the matlab package 'Kernel Two Sample Test'. We are grateful to Maria Rizzo and Gabriel Szekely who developed and maintain the 'energy' package and Arthur Gretton who developed and maintains the 'Kernel Two Sample Test' package.

\section{REFERENCES}

[1] A. H. Baker, D. M. Hammerling, M. N. Levy, H. Xu, J. M. Dennis, B. E. Eaton, J. Edwards, C. Hannay, S. A. Mickelson, R. B. Neale, D. Nychka, J. Shollenberger, J. Tribbia, M. Vertenstein, and D. Williamson. 2015. A new ensemble-based consistency test for the Community Earth System Model (pyCECT v1.0). Geoscientific Model Development 8, 9 (2015), 2829-2840. https://doi.org/10.5194/gmd-8-2829-2015

[2] E3SM Project. 2018. Energy Exascale Earth System Model (E3SM). [Computer Software] https://dx.doi.org/10.11578/E3SM/dc.20180418.36. https://doi.org/10.
11578/E3SM/dc.20180418.36

[3] Pierre Gentine, Mike Pritchard, Stephan Rasp, Gael Reinaudi, and Galen Yacalis. 2018. Could machine learning break the convection parameterization deadlock? Geophysical Research Letters 45, 11 (2018), 5742-5751.

[4] Chris Golaz and co authors. 2019. The DOE E3SM coupled model version 1: Overview and evaluation at standard resolution. Journal of Advances in Modeling of Earth Systems (accepted) (2019).

[5] Arthur Gretton, Karsten M. Borgwardt, Malte J. Rasch, Bernhard Schölkopf, and Alexander Smola. 2012. A Kernel Two-sample Test. F. Mach. Learn. Res. 13, 1 (March 2012), 723-773. http://dl.acm.org/citation.cfm?id=2503308.2188410

[6] Joseph H. Kennedy, Salil Mahajan, and Katherine J. Evans. 2018. LIVVkit/evv4esm: EVV 0.1.1. https://doi.org/10.5281/zenodo. 2541244

[7] Salil Mahajan, Katherine J. Evans, Joe Kennedy, Min Xu, Matt R. Norman, and Marcia L. Branstetter. 2019. Ongoing solution reproducibility of earth system models as they progress toward exascale computing. International fournal of High Performance Computing Applications (in press) (2019).

[8] Salil Mahajan, Abigail L. Gaddis, Katherine J. Evans, and Matthew R. Norman. 2017. Exploring an Ensemble-Based Approach to Atmospheric Climate Modeling and Testing at Scale. Procedia Computer Science 108 (2017), 735 - 744. https: //doi.org/10.1016/j.procs.2017.05.259 International Conference on Computational Science, ICCS 2017, 12-14 June 2017, Zurich, Switzerland.

[9] F. Massonnet, M. Ménégoz, M. C. Acosta, X. Yepes-Arbós, E. Exarchou, and F.J. Doblas-Reyes. 2018. Reproducibility of an Earth System Model under a change in computing environment. Technical Report. Barcelona Supercomputing Center.

[10] Nvidia 2018. PGI compiler and tools: User's guide for x86-64 CPUs. Nvidia.

[11] Yun Qian, Huiping Yan, Zhangshuan Hou, Gardar Johannesson, Stephen Klein, Donald Lucas, Richard Neale, Philip Rasch, Laura Swiler, John Tannahill, Hailong Wang, Minghuai Wang, and Chun Zhao. 2015. Parametric sensitivity analysis of precipitation at global and local scales in the Community Atmosphere Model CAM5. Journal of Advances in Modeling Earth Systems 7, 2 (2015), 382-411. https://doi.org/10.1002/2014MS000354

[12] Paul R. Rosenbaum. 2005. An exact distribution-free test comparing two multivariate distributions based on adjacency. Journal of the Royal Statistical Society: Series B (Statistical Methodology) 67, 4 (2005), 515-530. https: //doi.org/10.1111/j.1467-9868.2005.00513.x

[13] J. Rosinski and D. Williamson. 1997. The Accumulation of Rounding Errors and Port Validation for Global Atmospheric Models. SIAM fournal on Scientific Computing 18, 2 (1997), 552-564. https://doi.org/10.1137/S1064827594275534

[14] Gábor J Székely and Maria L Rizzo. 2004. Testing for equal distributions in high dimension. InterStat 5 (2004), 1249-1272.

[15] H. Wan, K. Zhang, P. J. Rasch, B. Singh, X. Chen, and J. Edwards. 2017. A new and inexpensive non-bit-for-bit solution reproducibility test based on time step convergence (TSC1.0). Geoscientific Model Development 10, 2 (2017), 537-552. https://doi.org/10.5194/gmd-10-537-2017 
a.

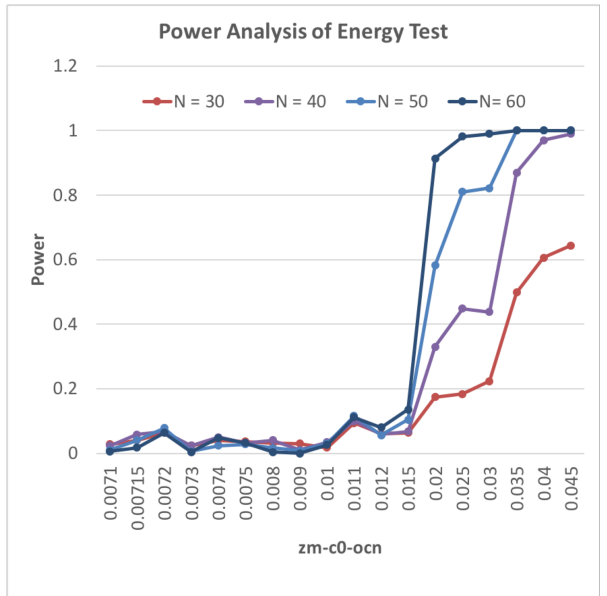

b.

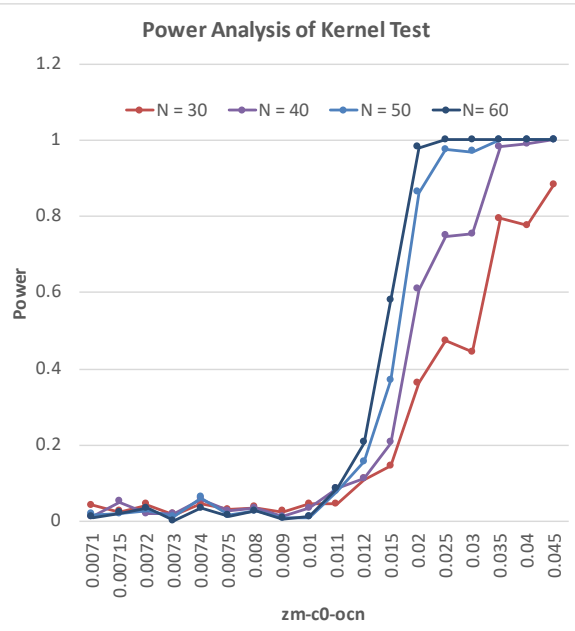

C.

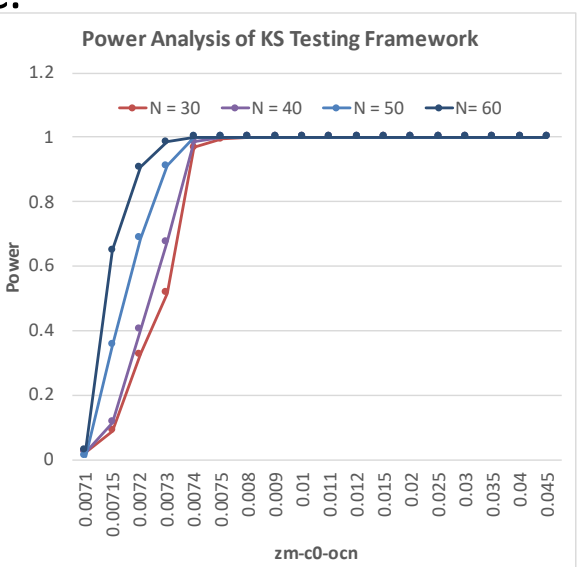

Figure 1: Power Analysis of the three tests for the zm_c0_ocn experiment set (ZM_SET): (a) energy test, (b) kernel test and (c) KS testing framework. Empirically (random sampling) estimated statistical Power $(1-\beta)$ - probability of rejecting a false null hypothesis - of the test at the $95 \%$ confidence level for different ensemble sizes $(N=30,40,50,60)$. The null hypothesis that two simulation ensembles are statistically identical is tested for each perturbed zm_c0_ocn case against the $\mathrm{zm}$.c0_ocn $=0.007$ case. Figure 1c is reproduced from Mahajan et al. [7]. 


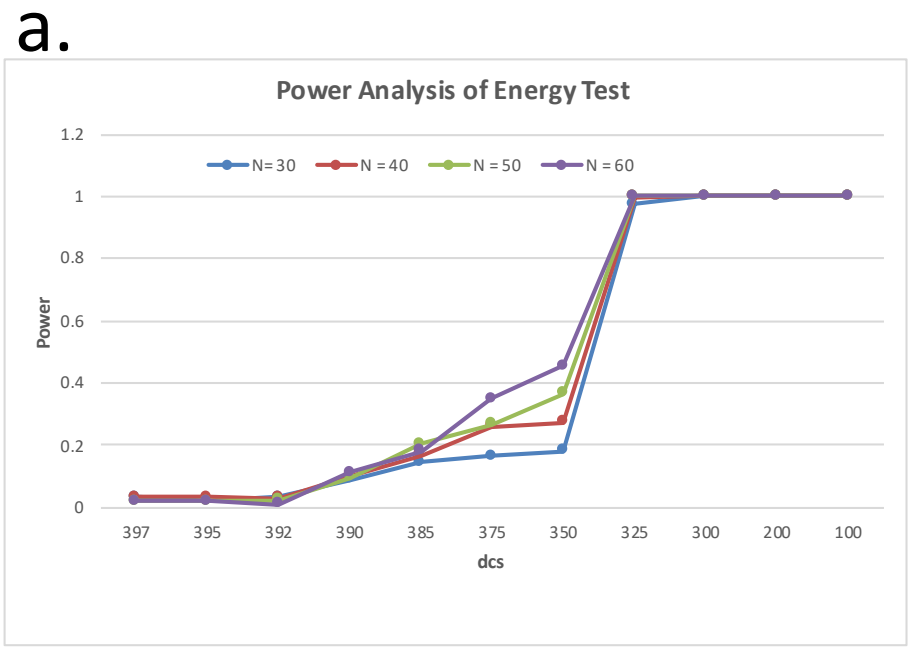

b.

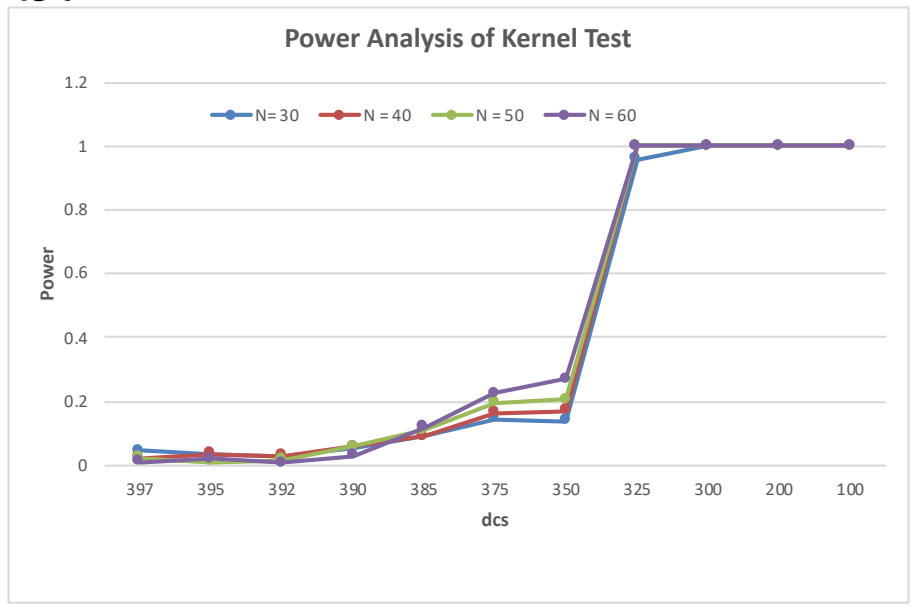

C.

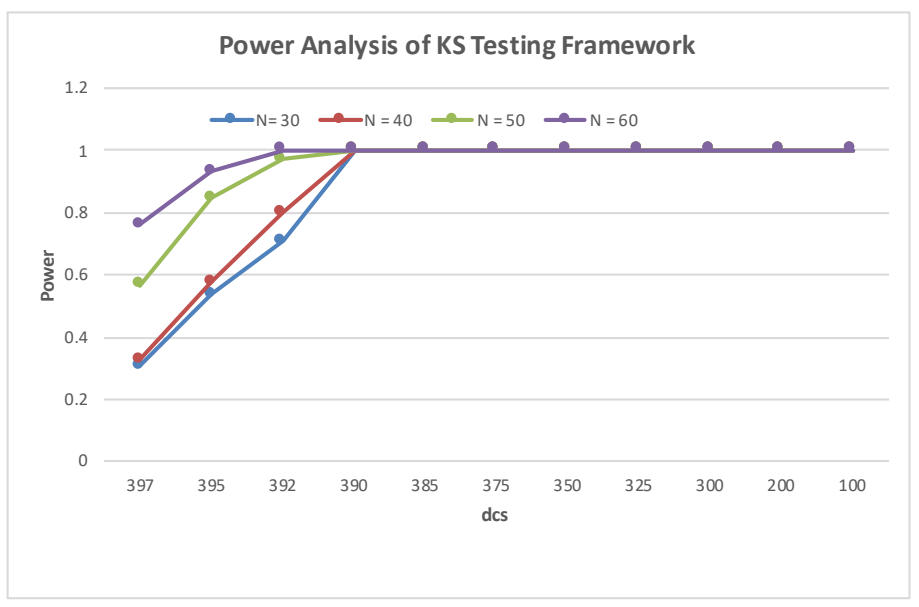

Figure 2: Power Analysis of the three tests for the $d c s$ experiment set (MICRO_SET): (a) energy test, (b) kernel test and (c) KS testing framework. Empirically (random sampling) estimated statistical Power $(1-\beta)$ - probability of rejecting a false null hypothesis - of the test at the $95 \%$ confidence level for different ensemble sizes $(N=30,40,50,60)$. The null hypothesis that two simulation ensembles are statistically identical is tested for each perturbed $d c s$ case against the $d c s=400.0$ case. 

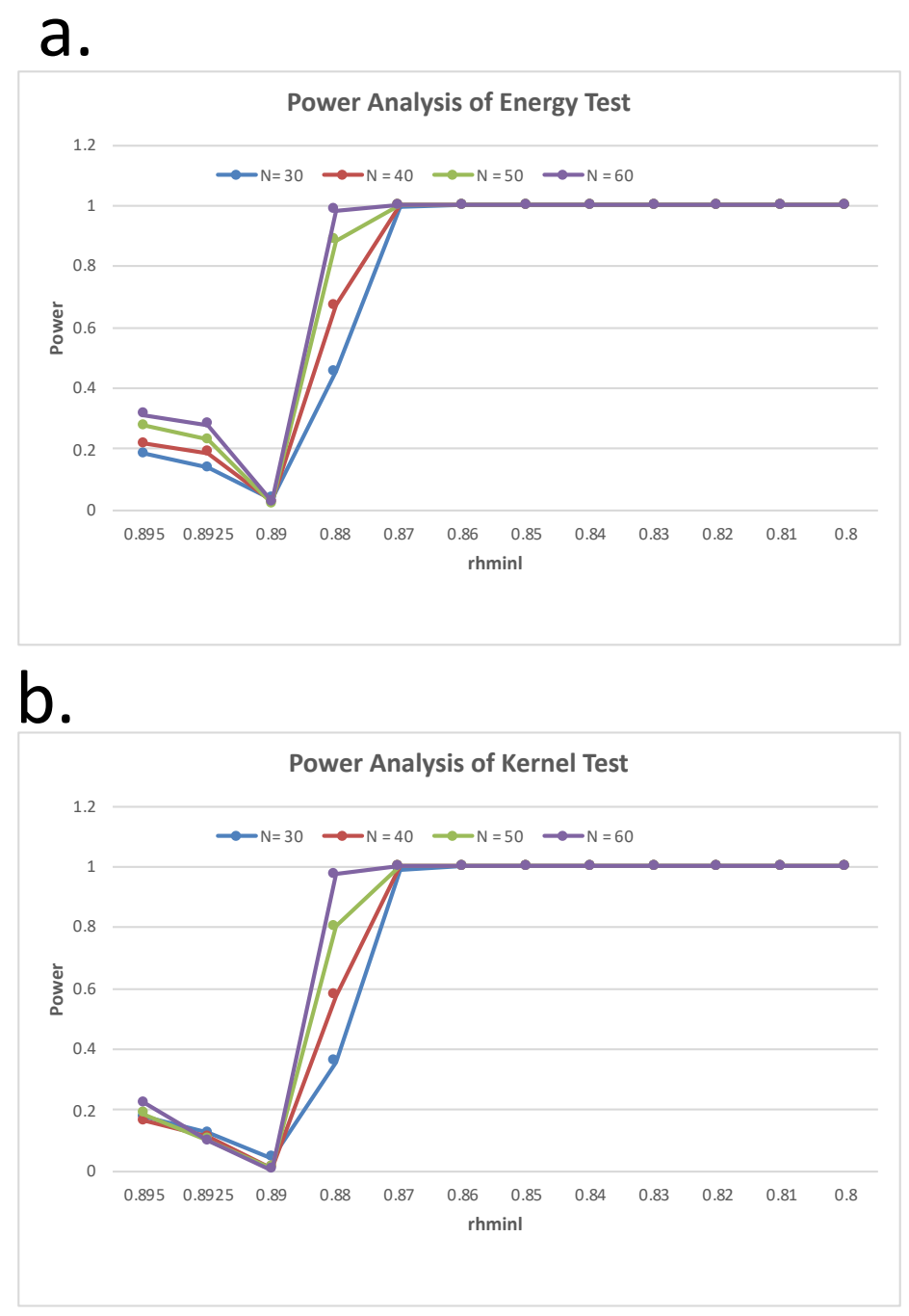

\section{C.}

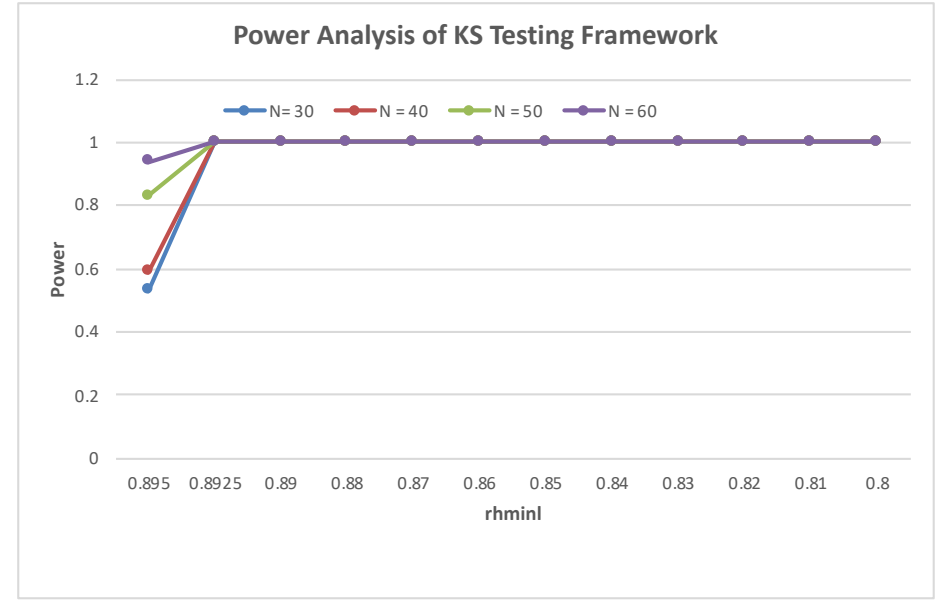

Figure 3: Power Analysis of the three tests for the rhminl experiment set (MACRO_SET): (a) energy test, (b) kernel test and (c) KS testing framework. Empirically (random sampling) estimated statistical Power $(1-\beta)$ - probability of rejecting a false null hypothesis - of the test at the $95 \%$ confidence level for different ensemble sizes $(N=30,40,50,60)$. The null hypothesis that two simulation ensembles are statistically identical is tested for each perturbed $\mathrm{rhminl}$ case against the control $\mathrm{rhminl}=0.8975$ case. 